\title{
VALOR ACTUAL DE LA BIOPSIA DE VESÍCULAS SEMINALES EN PACIENTES CON CÁNCER DE PRÓSTATA E INFLUENCIA DE LA PROSTATECTOMÍA RADICAL EN PACIENTES CON INFILTARCIÓN SEMINAL.
}

\author{
Carlos Alberto Allepuz Losa, Ángel Borque Fernando, Vicente Andrés Lázaro', Francesc Filipo \\ Berlanga², Patricia Serrano Frago, Marta Allúe López, María Jesús Gil Sanz, Pedro Gil Martínez \\ y Luis Angel Rioja Sanz.
}

Servicio de Urología. Hospital Universitario "Miguel Servet". Zaragoza. España.

'Servicio de Urología. Hospital de Alcañiz. Tervel. España.

2Servicio de Anatomía Patológica. Hospital Universitario "Miguel Servet". Zaragoza. España.

\begin{abstract}
Resumen.- OBJETIVO: Comprobar si la prostatectomía radical puede influir favorablemente en la supervivencia cáncer-específica (SCE), tiempo libre de hormonorresistencia y tiempo libre de metástasis (TLM) y calidad de vida (CV), en pacientes con adenocarcinoma de próstata e invasión de vesículas seminales, asi como, realizar una actualización de nuestro pensamiento sobre la biopsia de vesículas seminales.
\end{abstract}

MÉTODOS: Se incluyeron 114 pacientes En 46 se diagnosticó invasión de vesículas seminales tras la realización de prostatectomía radical (PRVS) de intención curativa y en 68 casos, la invasión fue diagnosticada mediante la biopsia de las mismas (BxVS) no practicándose la prostatectomía radical. Se compararon la SCE, tiempo hasta la hormonorresistencia desde el inicio del tratamiento hormonal (THR), TLM y CV medida en necesidades de atención hospitalaria en ambos grupos. La mediana del tiempo de seguimiento fue de 52,6 meses.

RESULTADOS: No hubo diferencias estadísticamente significativas entre ambos grupos en SCE, THR, TLM y CV. La SCE a 3 y 5 años fue del 100\% y $80.77 \%$ para el grupo PRVS y del $92.03 \%$ y $77,46 \%$ para el grupo BxVS. El THR a 3 y 5 años fue del $90.6 \%$ y $58.7 \%$ para el grupo PRVS y del $74,4 \%$ y $56,2 \%$ para el grupo $B \times V S$. En la regresión de Cox se mostraron predictores independientes de SCE el grado primario y suma de Gleason y de THR el estadio clínico.

CONCLUSIONES: La prostatectomía radical como monoterapia no influye de forma estadísticamente significativa en el tiempo de seguimiento realizado, en la SCE, THR, TLM y CV de los pacientes con cáncer de próstata e invasión de vesículas seminales asociada a otros factores de mal pronóstico (Gleason y PSA desfavorables). El valor de la biopsia de vesículas seminales permanece en el estudio de nuevos tratamientos multimodales, como pueden ser la quimioterapia en combinación con la cirugía, y esta pendiente de definir en la planificación de la radioterapia y la criocirugía.

Palabras clave: Cáncer de próstata. Estadificación. Biopsia. Vesículas seminales. Prostatectomía radical. Supervivencia.

Summary.- OBJECTIVES: To evaluate if radical prostatectomy may positively influence cancer-specific
Carlos Allepuz Losa Servicio de Urología Hospital Universitario Miguel Servet Pso. Isabel la Católica, 1 y 3 50009 Zaragoza. (España). callepuz@salud.aragon.es 
survival (CSS), hormone-resistance-free time, metastasisfree time, and quality of life(Qol) of patients with prostate adenocarcinoma and seminal vesicle invasion, and also to update our thoughts about seminal vesicle biopsy.

METHODS: 114 patients were included. Forty-six cases were diagnosed of seminal vesicle invasion after radical prostatectomy; 68 cases were diagnosed of seminal vesicle invasion after biopsy, not undergoing then surgery. Cancer specific survival, time to hormone resistance from the start of hormonal treatment, metastasis free time and QoL, measured as need for hospital care, were compared between groups. Median follow-up time was 52.6 mos.

RESULTS: There were not statistically significant differences between groups in CSS, time to hormone resistance, metastasis free time and QoL. Three and five-year cancer specific survival were $100 \%$ and $80.77 \%$ for the radical prostatectomy group and $74.4 \%$ and $56.2 \%$ for the biopsy group. Primary grade and Gleason Score were independent predictors for CSS in the Cox regression test; clinical stage was independent predictor for time to hormone resistance.

CONCLUSIONS: Radical prostatectomy as monotherapy does not show a statistically significant influence on follow-up time, CSS, time to hormone resistance, metastasis free time or QoL in patients with prostate cancer and seminal vesicle invasion associated with other bad prognostic factors (unfavourable Gleason and PSA). The value of seminal vesicle biopsy remains for the study of new multimodal treatments, such as chemotherapy + surgery, and it is to be defined in the planning of radio and cryosurgery.

Keywords: Prostate cancer. Staging. Biopsy. Seminal vesicles. Radical prostatectomy. Survival.

\section{INTRODUCCIÓN}

El cáncer de próstata es uno de los cánceres más frecuentes del mundo occidental. No siendo su distribución geográfica constante en todo el mundo, según la base de datos Globocan 2002, la mayor incidencia la registran los países más desarrollados siendo en ellos la tasa cruda y la tasa estandarizada por la edad 6 y 13 veces superior respectivamente, en relación a las de los países menos desarrollados. Dentro de Europa los países que presentan las tasas de incidencia más elevadas son Suecia y Suiza, siendo los países que presentan las tasas de mortalidad más elevadas Suecia y Noruega (1).

En EEUU representa el segundo tumor con mayor mortalidad, después del broncopulmonar. La probabilidad de desarrollar un nuevo cáncer a lo lar- go de la vida es de $17.81 \%$, es decir, 1 de cada 6 pacientes, siendo mayor dicha probabilidad a partir de los 60 años (2).

En España, según los datos del Instituto Nacional de Estadística, el cáncer de próstata es la segunda causa de defunción por tumor en varones, después del cáncer de bronquios y pulmón. En el año 2003, se registraron 5.606 defunciones con una edad media de 79,4 años, dos años más que los que fallecieron hace 10 años. La tasa de mortalidad fue de 27,179 por 100.000 varones de cualquier edad $(3,4)$.

Después de la incorporación del PSA, el estadio del tumor al diagnóstico ha cambiado, hallándose más casos órgano-confinados y menos metástasis a distancia (5). En la época pre-PSA sólo el $33 \%$ de los tumores detectados mediante tacto rectal eran patológicamente órgano-confinados, frente al $62-71 \%$ en la actualidad (6). Nuestro programa de diagnóstico precoz (7), basado en la utilización del PSA y tacto rectal y realizado en población referida desde una consulta médica, también ha confirmado estos hechos, de manera, que en 1991 el $48 \%$ de los pacientes con edad de 70 años o menor a los que se les practicó una prostatectomía radical derivada del diagnóstico de cáncer de próstata en este programa tenían el tumor órgano-confinado, frente al $62,5 \%$ en 1995, y lo que es más demostrativo, el volumen tumoral hallado en la pieza de prostatectomía descendió de 3,87 c.c. de mediana a 1,35 c.c. en 1995 y a 1,1 c.c. en el 2000.

Si bien, la prostatectomía radical es un tratamiento difundido para el tratamiento de próstata localizado, su efectividad esta limitada, entre otras cosas, por la infraestadificación. Dentro de la enfermedad no organo-confinada, esta justificada una especial reflexión a los pacientes con estadio patológico pT3, ya que clásicamente y hasta nuestros días, ha sido descrita una clara diferencia en cuanto a la supervivencia y recidiva local $y / 0$ a distancia tras prostatectomía entre los pacientes que presentan extensión extracapsular sin afectación de las vesículas seminales (T3a) y los que tienen invasión de las mismas (T3b) (8-14) de manera que podría considerarse que aquellos pacientes con invasión de vesículas seminales tienen una enfermedad diseminada, al tener una supervivencia similar a la de aquellos pacientes con infiltración de los ganglios linfáticos $(12,15)$. Para Ahlering y cols. (16) la presencia de invasión seminal supondría un factor de mal pronóstico en cuanto a recidiva bioquímica y aparición de metástasis incluso en pacientes con cáncer de próstata y afectación ganglionar sometidos a prostatectomía radical y deprivación hormonal precoz. 
Durante mucho tiempo, se ha intentado dilucidar el origen del mal pronóstico de la invasión de las vesículas seminales. Clásicamente se intentaba interpretar a través del conocimiento de que un $32-50 \%$ de los pacientes con invasión de vesículas seminales presentaban afectación linfática en la linfadenectomía habitual de estadificación (17). No obstante, de este hecho se derivaba la pregunta ¿por qué los pacientes con invasión aislada de vesículas seminales presentan también un mal pronóstico? siéndo la contestación más lógica: porque están mal estadificados (18). En lo que ya es una hipótesis clásica (19), cuando un adenocarcinoma de próstata ha iniciado la extensión local hacia las vesículas seminales en más de un 32-50\% (20) ya presentaría afectación de los ganglios linfáticos regionales al menos, eliminando las posibilidades de curación de la cirugía y ensombreciendo su pronóstico. Esta diseminación a los ganglios linfáticos puede discurrir no sólo por los ubicados en la fosa obturatriz (21) que es la zona habitualmente disecada en la linfadenectomía que acompaña a la prostatectomía radical sino también hacia otros ganglios del área pelviana (22-24) como entre otras las cadenas iliacas internas, iliacas externas y sacras, aunque hoy día no existen datos concluyentes que aconsejen la realización de la linfadenectomía sacra por su baja rentabilidad diagnóstica al considerarse un segundo escalón del drenaje linfático prostático (25) mientras que sí comienzan a existir trabajos que abogarían por la realización de la linfadenectomía extendida que incluyera los ganglios iliacos internos y externos $(24,26)$. Así mismo, con la intención de disminuir la morbilidad que conlleva la linfadenectomía extendida y disminuir su extensión se ha propuesto la búsqueda del ganglio centinela previamente marcado con tecnecio-99 $(21,22,27)$.

En directa relación con el ominoso pronóstico de la invasión seminal que no siempre se acompaña de constatación de afectación linfática regional se ha postulado que la invasión de los ganglios linfáticos periprostáticos o periseminales, habitualmente no evaluados en el estudio patológico, aún en ausencia de invasión de las cadenas linfáticas pelvianas debería considerarse un estadio avanzado equiparable al estadio pN1 (28).

Tras este planteamiento clásico se han publicado con posterioridad diversas series de pacientes prostatectomizados con estudio de su progresión en las que se expresa que el estadio pT3b siendo un factor de mal pronóstico debe ser estratificado según otras variables acompañantes de modo que la suma de este estadio con determinados factores desfavorables será la que predecirá la progresión tumoral. Los factores pronósticos que se han descrito en diferentes estudios como predictivos de forma independiente son $(13,29-41)$ :

- La suma de Gleason del espécimen de prostatectomía - El valor de psa preoperatorio

- La invasión seminal localizada versus la extensa

- La presencia de afectación de márgenes tumorales

- La invasión vascular en la pieza quirúrgica

- La ploidía del DNA

- La edad

- El volumen tumoral

- La coexistencia de afectación ganglionar

Por tanto, estos autores, al igual que nosotros, consideran el estadio pT3b como un factor de mal pronóstico pero que se puede estratificar en relación a la coexistencia de otros factores de mal pronóstico.

Todos estos hechos nos condujeron a plantear un proyecto de investigación cuyo objetivo era comprobar si la prostatectomía radical (PR) influía favorablemente en la supervivencia cáncer-específica (SCE), tiempo libre de hormonorresistencia y tiempo libre de metástasis y calidad de vida, medida ésta última en necesidades de atención hospitalaria (urgencias, ingresos e intervenciones) en pacientes con adenocarcinoma de próstata e invasión de vesículas seminales, como se describe a continuación.

\section{MATERIAL Y MÉTODOS}

Se incluyeron 114 pacientes tratados previamente al año 1.999. En 46 se diagnosticó invasión de vesículas seminales tras la realización de prostatectomía radical (PRVS) de intención curativa y en 68 casos, la invasión fue diagnosticada mediante la biopsia de las mismas (BxVS) no practicándose la prostatectomía radical.

Las biopsias de vesículas seminales se han realizado y analizado siguiendo la técnica previamente descrita por nosotros (42) y la prostatectomía radical se ha practicado por vía retropúbica de acuerdo a la descripción de la técnica según Walsh (43).

Se definió hormonorresistencia como el momento en el que una vez instaurado el bloqueo androgénico completo se produce una elevación del PSA.

Como variables resultado se consideraron fundamentalmente la supervivencia cáncer-específica, tiempo libre de hormonorresistencia y tiempo libre de metástasis en pacientes con adenocarcinoma de próstata e invasión de vesículas seminales y 
calidad de vida, medida ésta última en necesidades de atención hospitalaria (urgencias, ingresos e intervenciones).

Se utilizó para el análisis de los datos pruebas de significación estadística bivariantes paramétricas cuando la variable seguía una distribución normal y pruebas no paramétricas en el caso contrario, valorándose la normalidad mediante el test de Kolmogrov-Smirnov. Para determinar los niveles de significación estadística de las tablas de contingencia y pruebas no paramétricas se utilizó el método de Monte Carlo.

El análisis de supervivencia se realizó mediante el método de Kaplan-Meier, así como la prue- ba de log rank. La evaluación de los diferentes factores pronósticos y su relación con el tiempo se realizó utilizando el modelo de regresión de riesgos proporcionales de Cox.

El análisis estadístico se realizó mediante el programa SPSS 10.

\section{RESULTADOS}

Las características más relevantes de los 2 grupos de pacientes que se comparan, el grupo diagnosticado de infiltración seminal mediante biopsia, y el grupo diagnosticado mediante prostatectomía radical queda reflejado en la Tabla $\mathrm{I}$.

\section{TABLA I. ESTADÍSTICA DESCRIPTIVA POR GRUPOS DE COMPARACIÓN.}

\begin{tabular}{|c|c|c|c|}
\hline & Grupo Biopsia VVSS & Grupo P. Radical & Valor de $p$ \\
\hline Edad (años) & & & $0.048^{*}$ \\
\hline Media (IC 95) & $66.9(65.8-68)$ & $65.1(63.5-66.6)$ & \\
\hline Mediana (AQ) & $67.5(6)$ & $66.5(6.5)$ & \\
\hline PSA preoperatorio (ngr./ml.) & & & $0.0001^{* *}$ \\
\hline Media (IC 95) & $64.8(44.5-85.1)$ & $27.8(17-38.7)$ & \\
\hline Mediana (AQ) & $37.6(39.3)$ & $17.7(21.3)$ & \\
\hline Estadio Clínico $\mathrm{n} \cong(\%)$ & & & $0.101^{*}$ \\
\hline$\leq \mathrm{CT} 2$ & $43(63.2)$ & $36(78.3)$ & \\
\hline$>\mathrm{CT} 2$ & $25(36.8)$ & $10(21.7)$ & \\
\hline Gleason primario $\mathrm{n}(\%)$ & & & $0.23^{*}$ \\
\hline 1 & 0 & 0 & \\
\hline 2 & $3(4.4)$ & $5(7)$ & \\
\hline 3 & $24(35.3)$ & 19 (41.3) & \\
\hline 4 & $37(54.4)$ & $21(45.7)$ & \\
\hline 5 & $4(5.9)$ & $1(2.2)$ & \\
\hline Suma de Gleason $n^{\circ}(\%)$ & & & $0.077^{*}$ \\
\hline $2-4$ & $3(4.4)$ & $5(10.9)$ & \\
\hline $5-6$ & $26(38.2)$ & 19 (41.3) & \\
\hline 7 & $21(30.9)$ & $18(39.1)$ & \\
\hline $8-10$ & $18(26.45)$ & $4(8.7)$ & \\
\hline Tiempo de seguimiento (meses) & & & $0.034^{* *}$ \\
\hline Media (IC 95) & $59.6(53.2-66.1)$ & $51.4(43.3-59.6)$ & \\
\hline Mediana (AQ) & $57.4(44.3)$ & $41.8(32.1)$ & \\
\hline
\end{tabular}

Biopsia VVSS: Biopsia de vesículas seminales P. Radical: Prostatectomía Radical (IC 95): Intérvalo de confianza del 95\% A.Q.:Amplitud intercuartilar * t-test-Chi2 ** U de Mann-Whitney 
Sólo la edad, el PSA preoperatorio y el tiempo de seguimiento mostraron diferencias estadísticamente significativas entre grupos (pacientes con prostatectomía radical versus sólo con biopsia de vesículas seminales). Ni el estadio clínico considerado como localizado frente a localmente avanzado, ni el grado de Gleason considerado en su patrón primario, secundario o su suma, en diversas categorías mostraron diferencias significativas entre grupos. Tampoco lo hicieron la presencia o no de bloqueo hormonal neoadyuvante ni su duración. Para el conjunto de los pacientes la mediana de seguimiento fue de 52,6 (amplitud cuartilar: 39.7) meses y la media de 56.3 (IC95\%: 51.3-61.3) meses.

De los 114 pacientes, 37 llevaron tratamiento hormonal neoadyuvante, 25 pacientes en el grupo de Bx.VS con una mediana de 3.5 meses (amplitud cuartilar de 3.7) y 12 en el grupo de PRVS con una mediana de 5 meses (amplitud cuartilar de 4). Tras la intervención, 100 pacientes fueron sometidos a tratamiento hormonal continuado hasta llegar a la hormonorresistencia, 66 en el grupo de Bx.VS y 34 en el grupo de PRVS. En todos los casos la $\mathrm{p}$ fue mayor de 0.05 .
La supervivencia media cáncer específica (Figura 1) fue de 92.8 meses (IC95\%: 84.4-101.2) en pacientes con PRVS frente a 106.1 meses (IC95\%: 92.2-120.1) en pacientes con BxVS (LogRank $p=0.46$ ). Los fallecimientos por cáncer ocurridos fueron $15(22,1 \%)$ en el grupo de Bx.VS y $6(13,1 \%)$ en el de PRVS.

El tiempo hasta la hormonorresistencia desde el inicio del tratamiento hormonal (neoadyuvante + adyuvante) (Figura 2) no fue significativamente diferente en ambos grupos (LogRank $p=0.67$ ), siendo la media de 69.7 (IC95\%: 60.2-79.3) y 57.4 (IC95\%: 50.4-64.4) meses, en pacientes con BxVS y PRVS respectivamente, y la mediana de 66.9 meses en ambos grupos. Dentro del grupo de BxVS el $48.5 \%(32 / 66)$ llegaron a ser hormonorresistentes, y el 20,6\% (7/34) de los del grupo a los que se les practicó una prostatectomía radical.

Veintidos pacientes del grupo BxVS y 9 del grupo PRVS presentaron metástasis lóseas -30 casoso linfáticas -1 caso-). No se encontraron diferencias estadísticamente significativas en el tiempo hasta su diagnóstico entre ambos grupos (LogRank $p=0.4$ ),

TABLA II. PROBABILIDAD A 3 Y 5 AÑOS DE SUPERVIVENCIA CÁNCER ESPECÍFICA, HORMONOSENSIBILIDAD DESDE EL INICIO DEL TRATAMIENTO HORMONAL Y SUPERVIVENCIA LIBRE DE METÁSTASIS.

\section{Grupo Biopsia VVSS Grupo P. Radical}

Probabilidad de Supervivencia cáncer específica (\%)
A 3 años (IC 95)
$92(85.3-98.7)$
100
A 5 años (IC 95)
$77.5(66-89)$
$80.8(63.6-98)$

Probabilidad de Hormonosensibilidad desde el inicio del tratamiento hormonal (\%)
A 3 años (IC 95)
$74.4(63.5-85.3)$
$90.6(80.4-100)$
A 5 años (IC 95)
$56.2(42.5-70)$
$58.7(28.6-88.8)$

Probabilidad de Supervivencia libre de metástasis (\%)
A 3 años (IC 95)
$83.2(74.1-92.3)$
92.7 (84.8-100)
A 5 años (IC 95)
$71.1(59.3-83)$
$75.7(56.8-94.7)$

Biopsia VVSS: Biopsia de vesículas seminales P. Radical: Prostatectomía Radical (IC 95): Intérvalo de confianza del 95\% 


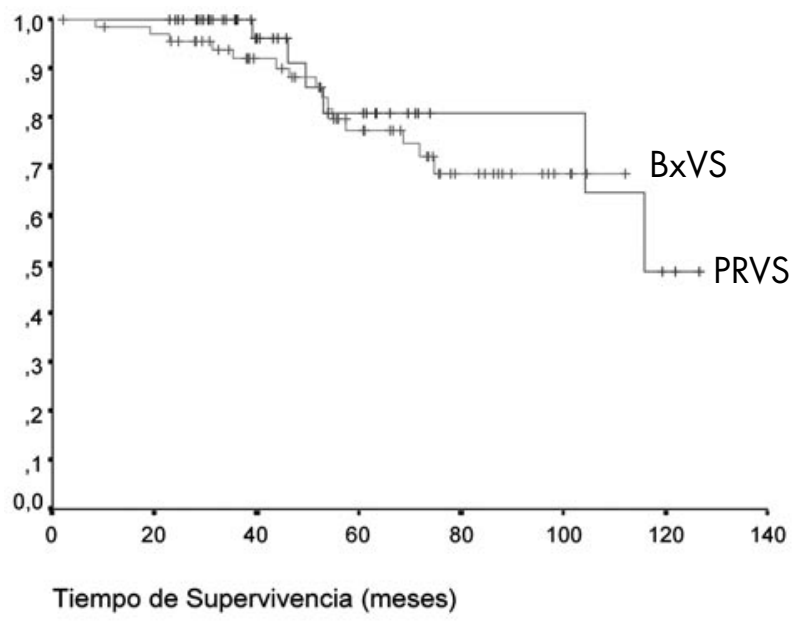

FIGURA 1. Supervivencia cáncer específica tras biopsia de vesículas seminales (BxVS) versus prostatectomía radical (PRVS).

siendo la siendo la media de 77.7 (IC95\%: 68.686.8) y 87.6 (IC95\%: 66.3-108.9) meses, en pacientes con BxVS y PRVS respectivamente, y la mediana de 101.8 meses en el grupo PRVS, no habiendo sido alcanzada ésta en el grupo de BxVS.

En la Tabla Il queda reflejada la probabilidad de supervivencia cáncer específica, de ser hormono-

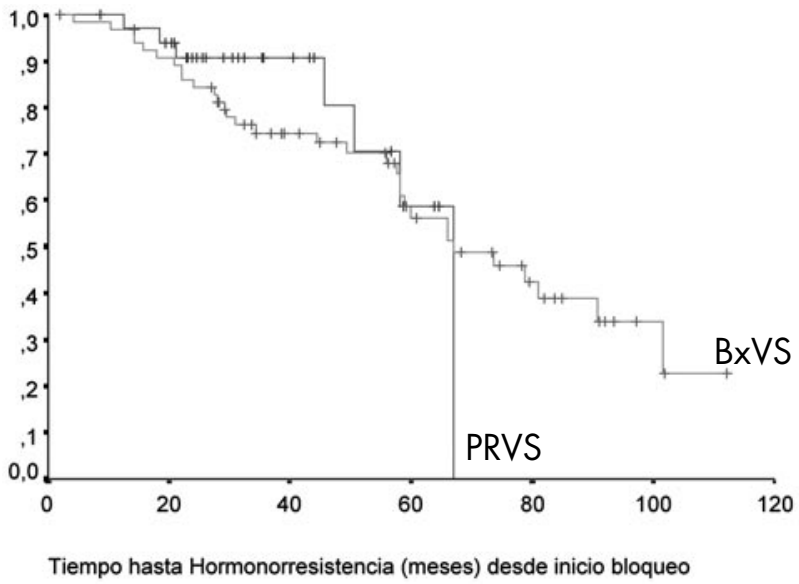

FIGURA 2. Tiempo hasta la hormonorresistencia desde el inicio del tratamiento en pacientes diagnosticados tras biopsia de vesículas seminales (BxVS) versus prostatectomía radical (PRVS).

sensible desde el inicio del tratamiento hormonal y supervivencia libre de metástasis a 3 y 5 años.

En relación a la calidad de vida, 36 pacientes sufrieron complicaciones evolutivas, lo cual supone un $31,6 \%$ del total de pT3b: 26 del grupo de BxVS (un $38,2 \%$ de este grupo de pacientes) y 10 del grupo de PRVS (un 21,7\% de este grupo) $p=0,0098$.

TABLA III. ANÁLISIS MULTIVARIANTE (REGRESIÓN DE COX). FACTORES PREDICTIVOS DE SUPERVIVENCIA CÁNCER ESPECÍFICA.

\begin{tabular}{|lll|}
\hline Variables predictivas & \multicolumn{2}{c|}{ SUPERVIVENCIA por CaP. } \\
\hline Modelo I: & Chi $^{2}$ & p \\
- Edad & 1,047 & N.S. \\
- Intervención Quirúrgica & 0,056 & N.S. \\
- PSA en categorías & 5,634 & N.S. \\
- Gleason primario, en categorías & 14,716 & $\mathbf{0 , 0 0 2}$ \\
- Estadio clínico (dicotómica) & 1,563 & N.S. \\
- Tto. Hormonal Adyuvante & 0,593 & N.S. \\
Modelo Il: & & \\
- Edad & 0,381 & N.S. \\
- Intervención Quirúrgica & 0,049 & N.S. \\
- Suma de Gleason, en 4 categorías & 4,764 & N.S. \\
- Estadio clínico (dicotómica) & 14,312 & 0,010 \\
- Tto. Hormonal Adyuvante & 2,335 & N.S. \\
\hline CaP.: Cáncer de Próstata & 0,146 & N.S. \\
N.S.: No Significación estadística & Tto.: Tratamiento \\
\hline
\end{tabular}


Siendo el número total de complicaciones de 86, 62 en el grupo BxVS y de 24 en el grupo sometido a prostatectomía radical.

De las 68 BxVS, 17 (25\%) precisaron una RTU de próstata en el seguimiento, $5(7,3 \%)$ precisaron una nefrostomía percutánea en el seguimiento, de ellos 2 continúan con cambios sucesivos e nefrostomía (uno con 1 y otro con 4). De las 46 prostatectomías radicales, $5(9,8 \%)$ precisaron una intervención por estenosis de la anastomosis en el seguimiento. De los 24 casos que precisaron intervención quirúrgica $17 / 18(94,4 \%)$ con BxVS y $4 / 6(66,6 \%)$ de PRVS necesitaron de anestesia general $\circ$ regional $(p=0,2867)$.

No hubo diferencias estadísticamente significativas entre grupos en cuanto a $\mathrm{n}^{\circ}$ total de complicaciones, $\mathrm{n}^{\circ}$ de intervenciones ambulantes, $\mathrm{n}^{\circ}$ de intervenciones con ingreso hospitalario, $\mathrm{n}^{\circ}$ de intervenciones que precisaron anestesia general, si bien fue próximo a la significación respecto al número total de complicaciones $(p=0.06$ con IC99\% de 0.06 0.07 con la Significación de Monte Carlo de la prueba de U de Mann-Withney).

Se diseñaron para cada una de las dos predicciones a estimar, supervivencia cáncer específica y tiempo hasta la hormonorresistencia, los modelos resultantes óptimos de análisis multivariante. Los resultados de este análisis multivariante por regresión de Cox se muestran en la Tabla III para la supervivencia cáncer específica y en la Tabla IV para el tiempo hasta la hormonorresistencia desde el inicio del tratamiento hormonal. Sólo el grado primario de Glea- son y su suma fueron predictores estadísticamente significativos de supervivencia cáncer específica, y el estadio clínico fue el único predictor estadísticamente significativo para tiempo hasta la hormonorresistencia desde el inicio del tratamiento hormonal.

\section{DISCUSIÓN}

El intervalo etario en el que se mueve nuestro grupo total de pacientes es bastante limitado, entre los 45,5 y los 76,5 años ( 31 años). Lo cierto es que, tanto las medianas como las medias están muy próximas entre el grupo de Bx.VS y el de PRVS, si bien la comparación de éstas mediante la prueba + llega a alcanzar significación estadística aunque en el límite $(p=0,048)$, nosotros no hemos estimado de interés clínico esta diferencia ni encontramos referentes en la literatura a este respecto.

Las diferencias observadas en el PSA preoperatorio entre los dos grupos de comparación y que alcanzan significación estadística $(p<0,01)$, se debería a la pre-selección de pacientes con factores de peor pronóstico en el grupo de Bx.VS, ya que la indicación para realizar una biopsia de vesículas seminales era precisamente la existencia de factores de mal pronóstico como son presentar un estadio clínico (TNM 1997) igual o superior a T2b (hasta 1997) o T3 (a partir de 1997) o independientemente la presencia de un PSA superior a $20 \mathrm{ngr} / \mathrm{ml}$. y/o una suma de Gleason de 7 (44-46). Además, no hay que olvidar que un porcentaje no desdeñable de pT3b procedentes de prostatectomía radical habían sido sometidos previamente a biopsia de vesículas seminales (21 pa-

TABLA IV. ANÁLISIS MULTIVARIANTE (REGRESIÓN DE COX). FACTORES PREDICTIVOS DE TIEMPO HASTA ALCANZAR LA HORMONORRESISTENCIA DESDE EL INICIO DEL TRATAMIENTO HORMONAL.

\begin{tabular}{|ccc|}
\hline Variables predictivas & $\begin{array}{c}\text { To hasta hormonorresistencia desde su inicio } \\
\mathbf{C h i}^{2}\end{array}$ & $\mathbf{p}$ \\
\hline Modelo I: & & \\
- Edad & 0,001 & N.S. \\
- Log PSA & 3,575 & N.S. \\
- Estadio clínico (dicotómica) & $\mathbf{4 , 2 1 4}$ & $\mathbf{0 , 0 4}$ \\
- Suma de Gleason, 4 categorías & 0,759 & N.S. \\
- Intervención Quirúrgica & 0,658 & N.S. \\
\hline
\end{tabular}

CaP.: Cáncer de Próstata

Tto.: Tratamiento

N.S.: No Significación estadística 
cientes de 46-45,6\%-) por poseer factores clínicos de mal pronóstico pero en los que la técnica había sido incapaz de diagnosticar la afectación seminal.

La comparación estadística del tiempo de seguimiento de ambos grupos muestra diferencias estadísticamente significativas $(p=0,034)$ entre los dos grupos con un mayor seguimiento de los pacientes con Bx.VS. La explicación clínica que encontramos a este hecho se basa en que hasta 1997 se biopsiaba a todos los pacientes con estadio clínico cT2b independientemente del PSA preoperatorio $y / o$ grado de Gleason, además en esta serie histórica la mayoría de los pacientes $\mathrm{pT} 3 \mathrm{~b}$ eran diagnosticados por biopsia de vesículas seminales por una mayor eficacia de la técnica, de ahí que el tiempo de seguimiento sea mayor partiendo de estos pacientes.

Las curvas de supervivencia cáncer específica de los pacientes $\mathrm{pT} 3 \mathrm{~b}$ según pertenezcan al grupo de la Bx.VS o al de la PRVS, nos muestran la inexistencia de diferencias estadísticamente significativas entre ambos grupos $(p=0,46)$, es decir que la probabilidad de morir por cáncer es muy similar, entre los pacientes $\mathrm{pT} 3 \mathrm{~b}$ con o sin exéresis de las vesículas seminales, lo cual no justificaría desde un punto de vista de supervivencia cáncer específica la realización de la prostatectomía radical como monoterapia ante la existencia de invasión seminal.

Nuestro estudio también mostró que no existían diferencias estadísticamente significativas entre el tiempo en el que se hacían hormonorresistentes los pacientes tratados que se les ha practicado biopsia de vesículas seminales o prostatectomía radical, es decir, el tipo de intervención que reciben los pacientes no influía en el tiempo de duración de la hormonosensibilidad a tratamientos de deprivación androgénica de primera línea. No obstante, de todo ello se deduce que la prostatectomía radical en pacientes pT3b aunque no influya en alargar el tiempo de eficacia del tratamiento hormonal quizá permita demorar su inicio. Demora que estaría indicada siempre y cuando se considere que el tratamiento hormonal diferido sea oncológicamente adecuado. Es preciso la realización de estudios con un mayor tiempo de seguimiento que confirmen estos hechos.

No se encontraron diferencias estadísticamente significativas en la supervivencia libre de metástasis entre ambos grupos. No obstante, este hecho debe de ser tomado con precaución pues como vemos en el grupo de BxVS, 22 pacientes $(32,3 \%)$ presentaron metástasis, haciéndolo a una media de 77,7 meses sobre un tiempo de seguimiento medio para este grupo de 59,6 meses, mientras que para el grupo de P.R. con 9 pacientes con metástasis $(19,6 \%)$ su aparición ocurrió a una media de 87,6 meses sobre una media de tiempo de seguimiento de 51,4 meses. Así las cosas, sería presumible que con un seguimiento mas amplio encontráramos lógicamente mas pacientes con metástasis, que previsiblemente provinieran en su mayoría del grupo de BxVS de mantenerse la tendencia a 3 y 5 años, lo que podría arrojar diferencias estadísticamente significativas a mayor seguimiento en la aparición de metástasis.

En la evaluación de la calidad de vida a través de las complicaciones evolutivas no se demostró que la realización de la prostatectomía radical supusiera un carácter paliativo en los pacientes con invasión seminal pues su comparación con estos mismos pacientes detectados por biopsia seminal no mostró un menor número de complicaciones, independientemente del tipo, en su evolución.

Dentro de los propósitos de este proyecto cuales eran estratificar a los pacientes con afectación de vesículas seminales en lo que a su pronóstico se refiere, vemos como al menos durante el tiempo de seguimiento evaluado la única variable que en el análisis multivariante fue capaz de incidir en la estimación de la supervivencia cáncer específica de estos pacientes sería el Gleason de la biopsia, con diferente significación según la forma en que se estime. En la pretensión de conocer si la prostatectomía radical podía tener interés paliativo en los pacientes con invasión seminal, vemos como, tanto en el análisis multivariante la variable "intervención quirúrgica" fue desechada en la estimación de la supervivencia cáncer específica, si bien es cierto que el número de fallecimientos por cáncer ocurridos no permitió sacar conclusiones a más largo plazo.

El análisis multivariante con respecto a la variable tiempo hasta la hormonorresistencia desde que se inició el tratamiento hormonal reveló como de todas las variables evaluadas las únicas que mostraron significación estadística en la estimación del tiempo hasta la hormonorresistencia era el estadio clínico en forma dicotómica, lo que podría ser una referencia a la situación de carga tumoral que el paciente tuviera, ya que eliminada esta variable, era el PSA preoperatorio en su forma logarítmica el que cobraba significación estadística (datos no mostrados), no siendo en ninguno de los casos la variable "intervención quirúrgica" (en referencia a la prostatectomía radical) una variable que presentara significación estadística.

Estos hallazgos coincidirían en parte con los obtenidos por otros autores. Así, Tefilli y cols. (29) describen cómo en un análisis multivariante la progresión tumoral de los pT3b vendría condicionada por la existencia de márgenes quirúrgicos, una suma 
de Gleason del espécimen $>7$, y/o un PSA preoperatorio > $10 \mathrm{ng} . / \mathrm{ml}$. coincidiendo con los trabajos de D'Amico y cols. (13) y de Debras y cols. (30) al encontrar que en un análisis multivariante de la progresión de los pacientes pT3b los factores que modifican la existencia de esta progresión son la invasión seminal localizada versus extensa, seguida de la suma de Gleason del espécimen; no siendo factores predictores de progresión la bilateralidad en la afectación seminal, el volumen tumoral, la extensión extracapsular o la afectación de márgenes. Epstein y cols. (31) citan un $30-40 \%$ de posibles curaciones en pacientes pT3bN0 tras cirugía sobre un total de 7 series revisadas con unos índices de no progresión a 5 años que oscilan entre el 5 y el $60 \%$ (mediana $40 \%$ ) $(29,30,32-35)$ por ello pretenden estratificar a los pacientes $\mathrm{pT} 3 \mathrm{~b}$ según factores pronósticos para seleccionar aquéllos potencialmente curables y encuentran que dentro del inherente mal pronóstico del pT3b éste se ve modificado en el análisis multivariante en función de diferentes combinaciones de Gleason, la afectación de márgenes y la invasión vascular del espécimen de prostatectomía, y rechazan como factores pronósticos la distancia que la invasión tumoral ha alcanzado a lo largo de la vesícula seminal, su vía de invasión (conductos eyaculadores, extraprostática, o metástasis) y otras medidas cuantificadoras de la extensión seminal o extraprostática; resultados muy similares a los descritos a nivel nacional por Zudaire y cols. (36) y Roberts y cols. (37), los cuales identifican como predictores de recidiva bioquímica en análisis univariante la existencia de ganglios linfáticos positivos, extensión extraprostática, afectación seminal, existencia de márgenes quirúrgicos positivos, el grado de Gleason de la pieza, el estadio clínico, el PSA preoperatorio y la edad; mientras que en el análisis multivariante los únicos factores predictores de recidiva que se mantienen son ganglios linfáticos positivos, afectación seminal, existencia de márgenes quirúrgicos positivos, y el grado de Gleason de la pieza. De modo similar, Blute y cols. (38) generan un modelo predictivo de progresión tras prostatectomía radical mediante regresión de Cox en el que las variables predictoras de progresión en un paciente pT3b serían la suma de Gleason de la pieza, el PSA preoperatorio, la existencia de márgenes positivos, la invasión seminal, y la hormonoterapia o radioterapia adyuvante, rechazando como factores predictores la extensión extracapsular o la ploidía del DNA. Lau y cols. (39) encuentran como factores predictores de progresión la invasión seminal, el PSA preoperatorio y la ploidía del DNA, sin que el patrón de Gleason primario aporte información adicional en un análisis multivariante. Sofer y cols. (40) encuentran que los factores pronósticos de recidiva tras prostatectomía radical en pacientes con invasión de vesículas seminales son la edad, un PSA mayor de $10 \mathrm{ngr} . / \mathrm{ml}$. y un volumen tumoral superior al $20 \%$. Bloom y cols. (41) consideran factores pronósticos de recidiva en pacientes con invasión de vesículas seminales tras prostatectomía radical la presencia de márgenes, el PSA preoperatorio y la suma de Gleason de la pieza de prostatectomía.

Como vemos, son diseños similares al realizado por nosotros pero en absoluto comparables dado que lo que en su mayoría la variable respuesta principal es la recidiva bioquímica y no la supervivencia cáncer específica ni el tiempo hasta la hormonorresistencia como es nuestro caso, tampoco se trata de pacientes $\mathrm{pT} 3 \mathrm{~b}$ en su totalidad sino del resultado tras prostatectomía radical.

Kasibhatla y cols. (47) realizan una amplia revisión de la literatura, y además de confirmar en ésta y en su experiencia el mal pronóstico de la infiltración seminal ponen de manifiesto que la cirugía no suele ser habitualmente curativa, y que hasta la fecha no se conoce todavía cual es el mejor tratamiento de los pacientes tras prostatectomía radical. Si se reconoce, que tanto el tratamiento postoperatorio con los tratamientos clásicos, hormonal, radioterapia o la suma de ambas debe ser evaluada prospectivamente.

Por tanto, de nuestro estudio se desprendió la clara conclusión de que no existían diferencias evolutivas estadísticamente significativas entre pacientes con BxVS y PRVS que permitieran sustentar la realización generalizada de una prostatectomía radical con carácter paliativo en monoterapia, en pacientes con adenocarcinoma de próstata con invasión de vesículas seminales en el periodo de seguimiento realizado, entendiendo por paliativo, aumento de la supervivencia cáncer específica, prolongación del tiempo de hormonosensiblidad, incremento periodo libre de metástasis o mejora de la calidad de vida medida en necesidades de atención hospitalaria (urgencias, ingresos e intervenciones).

No obstante, es preciso reconocer que nuestro estudio, aunque original y único en la literatura, tiene sus limitaciones, como es el hecho de que no fue ni prospectivo ni randomizado. Además, el perfil de los pacientes que en la actualidad se atienden en nuestro entorno son diferentes, como ya se ha comentado son pacientes con menos carga tumoral, menor psa y en etapas más precoces de la enfermedad. Así mismo, el tratamiento quirúrgico se aplica con una mayor precisión y experiencia, todo lo cual, podría ser motivo para que un estudio similar realizado en la actualidad con nuestra serie de pacientes más próximos y con un seguimiento más prolongado pudiera arrojar resultados diferentes. 


\section{La biopsia de vesículas seminales. Conceptos actuales}

Por tanto, si algo queda claro, tanto en nuestro estudio como en la literatura, es el mal pronóstico que conlleva la afectación de vesículas seminales cuando se asocia además con otros factores como son la indiferenciación celular o psa elevado. En la actualidad al cambiar el perfil del paciente están disminuyendo las indicaciones de biopsia de seminales en pacientes con indicación de cirugía radical de intención curativa en monoterapia, además y en este mismo sentido se abre el interrogante sobre la oportunidad de biopsiar a pacientes con Gleason 7, ya sea $3+4$ ó 4+3. Así mismo, el desarrollo de las técnicas de imagen puede en un futuro no muy lejano hacer disminuir en mayor medida, la necesidad de técnicas interventivas.

Otro aspecto diferente, es la prostatectomía radical enmarcada dentro de un esquema de tratamiento multimodal en pacientes de alto riesgo, por ejemplo asociada a quimioterapia (48). En estos casos, la biopsia de vesículas seminales podría tener poco papel en la práctica clínica en la actualidad desde un punto de vista terapéutico, puesto que el esquema esta en fase de estudio, pero si que podría tener un papel fundamental, desde el punto de vista investigacional, en la correcta estadificación de los pacientes, que permitiría verificar el verdadero papel de las terapias asociadas como la quimioterapia en la búsqueda de la tal ansiada infraestadificación terapéutica.

También se ha visto, que el resultado de la cirugía radical se pueden estratificar según posean o no los pacientes factores con mal pronóstico, como puede ser la suma de Gleason, en el sentido de que en algunos casos de alto grado (suma de Gleason 8) cuando el tumor es organoconfinado y el PSA preoperatorio intermedio (10-15 ngr/ml.), la cirugía radical proporciona un buen resultado a largo plazo $(49,50)$. En la búsqueda de estos pacientes la biopsia de vesículas seminales podría ser una opción válida.

La biopsia de vesículas seminales puede seguir teniendo un papel importante para planificar correctamente otro tipo de tratamientos, ya sea la radioterapia externa $(51,52)$, la braquiterapia (53) o inclusive la crioterapia $(54,55)$.

\section{CONCLUSIONES}

Nuestro trabajo de investigación revela que la prostatectomía radical como monoterapia no influye de forma estadísticamente significativa, en el tiempo de seguimiento estudiado, en la evolución (en relación a supervivencia cáncer específica, tiempo hasta la hormonorresistencia desde el inicio del tratamiento, tiempo libre de metástasis y calidad de vida) de los pacientes con cáncer de próstata e invasión de vesículas seminales asociada a otros factores de mal pronóstico (Gleason y PSA elevados). Hemos encontrado que el grado primario o suma de Gleason fueron predictores estadísticamente significativos de supervivencia cáncer específica y el estadio clínico fue un factor pronóstico independiente en estadísticamente significativo para tiempo hasta la hormonorresistencia desde el inicio del tratamiento hormonal.

Tanto la historia natural del cáncer de próstata, como sus posibilidades de estudio por la imagen y terapéuticas están cambiando. El rápido avance de las técnicas de imagen hace pensar que a corto o medio plazo, estas puedan sustituir, totalmente o en gran medida, a la biopsia de vesículas seminales en el estadificación del cáncer de próstata.

Además, éste cambio originará que la indicación de la biopsia de vesículas seminales disminuya para la selección de enfermos para tratamiento mediante prostatectomía radical como monoterapia, quedando probablemente su indicación limitada a aquellos casos de alto interés quirúrgico, con sumatorio de Gleason 8 o superior y/o un psa elevado (en nuestro ambiente actual de $20 \mathrm{ngr} . / \mathrm{ml}$.).

Es indudable que permanece su valor en el estudio y desarrollo de nuevos tratamientos multimodales, como pueden ser la quimioterapia en combinación con la cirugía.

El papel dentro de la planificación y racionalización de otros tratamientos como la radioterapia y la criocirugía puede ser prometedor, pero todavía esta pendiente de precisar.

\section{BIBLIOGRAFÍA y LECTURAS RECOMENDADAS (*lectura de interés $y^{* *}$ lectura fundamental)}

1. FERLAY, J.; BRAY, F.; PISANI, P. y cols.: "GLOBOCAN 2002. Cancer Incidence, Mortality and Prevalence Worldwide". IARC CancerBase No. 5, version 2.0. IARCPress, Lyon, 2004. Built Sep16-2005.

2. JEMAL, A.; MURRAY, T.; WARD, E. y cols.: "Cancer Statistics, 2005". CA Cancer J. Clin., 55: 10, 2005. 
3. INE.: "Defunciones según la Causa de Muerte 2003 Tasas de mortalidad por causas (lista reducida), sexo y edad". (Base de datos en Internet), Disponible en http://www.ine.es/inebase/ cgi/axi?AXIS_PATH=/inebase/temas/t15/p417/ a2003/10/\&FILE_AXIS=01004.px\&CGI_DEFAULT $=/$ inebase/temas/cgi.opt $\& C O M A N D O=S$ ELECCION\&CGI_URL=/inebase/cgi/

4. INE.: "Defunciones según la Causa de Muerte 2003. Nota de prensa de 24 de noviembre de 2005". Disponible en http://www.ine.esprensanp393.pdf.

5. FARKAS, A.; SCHENEIDER, D.; PERROTTI, M. y cols.: "National trends in the epidemiology of prostate cancer, 1973 to 1994: evidence for the effectiveness of prostate-specific antigen screening”. Urology, 52: 444, 1998.

6. SVETEC, D.: "Thompson I.M. PSA screening current controversy". Ann. Oncol., 9: 1283, 1998.

7. SANZ, J.I.; ALLEPUZ, C.; GIL, M.J. y cols.: "Diagnóstico precoz del cáncer de próstata. Análisis de 5 años”. Actas Urol. Esp., 21: 827, 1997.

8. MIDDLETON, R.G.; SMITH, J.A. Jr.; MELZER, R.B. y cols.: "Patient survival and local recurrence rate following radical prostatectomy for prostatic carcinoma". J. Urol., 136: 422, 1986.

9. PAULSON, D.F.; STONE, A.R.; WALTHER, P.J. y cols.: "Radical prostatectomy: anatomical predictors of sucess or failure". J. Urol., 136: 1041, 1986.

10. MUKAMEL, E.; DEKERNION, J.B.; HANNAH. y cols.: "The incidence and significance of seminal vesicle invasión in patients with adenocarcinoma of the prostate". Cancer, 59: 1535, 1987.

11. DEBRAS, B.; GUILLONNEAU, B.; BOUGARAN, J. y cols.: "Prognosis significance of seminal vesicle invasion on the radical prostatectomy specimen". Eur. Urol., 33: 271, 1998.

12. CATALONA, W.J.; SMITH, D.S.: "Cancer recurrence and survival rates after anatomic radical retropubic prostatectomy for prostate cancer: intermediate term results". J. Urol., 160: 2428, 1998.

13. D'AMICO, A.; WHITTINGTON, R.; MALKOWICZ, B. y cols.: "The combination of preoperative prostate specific antigen and postoperative pathological finfings to predict prostate specific antigen outcome in clinically localized prostate cancer". J. Urol., 160: 2096, 1998.

*14. GIL, P.; GIL, M.J.; ALLEPUZ, C. y cols.: "Cáncer de próstata estadio pT3 tras prostatectomía radical. Resultados en progresión y supervivencia”. Actas Urol. Esp., 24: 400, 2000.

**15. ROEHL, K.A.; HAN, M.; RAMOS, C.G. y cols.: "Cancer progression and survival rates following anatomical radical retropubic prostatectomy in 3.478 consecutive patients: long-term results". J. Urol., 172, 910, 2004

*16. AHLERING, T.E.; SKARECKY, D.W.; McLAREN, C.E. y cols.: "Seminal vesicle involvement in patients with D1 disease predicts early prostate specific antigen recurrence and metastasis after radical prostatectomy and early androgen ablation". Cancer, 94: 1648, 2002.

17. STONE, N.N.; STOCK, R.G.; PARIKH, D. y cols.: "Perineural invasion and seminal vesicle involvement predict pelvic lymph node metastasis in men with localized carcinoma of the prostate". J. Urol., 160: 1722, 1998.

**18. ALLEPUZ, C.; BORQUE, A.; RIOJA, C. y cols.: "Utilidad de la biopsia de vesículas seminales en el cáncer de próstata”. Tratado de Urología Oncológica, 2: 803, 2003.

19. GERVASI, L.A.; MATA, J.; EASLEY, J.D. y cols.: "Prognostic significance of lymph nodal metastases in prostate cancer". J. Urol., 142: 332, 1989.

20. STONE, N.N.; STOCK, R.G.; PARIKH, D. y cols.: "Perineural invasion and seminal vesicle involvement predict pelvic lymph node metastasis in men with localized carcinoma of the prostate". J. Urol., 160: 1722, 1998.

21. WEINGARTNER, K.; RAMASWAMY, A.; BITTINGER, A. y cols.: "Anatomical basis for pelvic lymphadenectomy in prostate cancer: result of an autopsy study and implications for the clinic". J. Urol., 156: 1969, 1996.

22. KOCJANCIC, E.; MONESI, G.; MINOCCI, D. y cols.: "The sentinel lymphnode in prostate cancer (pc), role of the radioimmunoguided surgery". Abstract 956 presentado en el 54th AUA Annual Meeting, June 2-7, 2001.

23. WAWROSCHEK, F.; VOGT, H.; WECKERMANN, D. y cols.: "Radioisotope guided pelvic lymph node dissection for prostate cancer". J. Urol., 166: 1715, 2001.

*24. HEIDENREICH, A.; ZOLTAN, V.; VON KNOBLOCH, R.: "Extended pelvis lymphadenectomy in patients undergoing radical prostatectomy: high incidence of lymph node metastasis". J. Urol., 167: 1681, 2002.

25. BRÖSSNER, C.; RINGHOFER, H.; HERNADY, T. y cols.: "Lymphatic drainage of prostatic transition and peripheral zones visualized on a three-dimensional workstation". Urology, 57: 389, 2001.

26. BURKHARD, F.C.; SCHUMACHER, M.; THALMANN, G.N. y cols.: "Is pelvic lymphadenectomy really necessary in patients with a serum prostate-specific antigen level of $<10 \mathrm{ng} / \mathrm{ml}$ undergoing radical prostatectomy for prostate cancer?". BJU Int., 95: 275, 2005.

27. CORVIN, S.; SCHILLING, D.; ANASTASIADIS, A.G. y cols.: "Radioisotope guided pelvic lymph node dissection for prostate cancer staging". Eur. Urol. Suppl., 4: 25, 2005.

28. KOTHARI, P.S.; SCARDINO, P.T.; OHORI, M. y cols.: "Incidence, location, and significance of periprostatic and periseminal vesicle lymph nodes in prostate cancer”. Am. J. Surg. Pathol., 25: 1429, 2001.

29. TEFILLI, M.V.; GHEILER, E.L.; TIGURET, R. y cols.: "Prognostic indicators in patiens with seminal vesicle involvement following radical prosta- 
tectomy for clinically localized prostate cancer". J. Urol., 160: 802, 1998.

30. DEBRAS, B.; GUILLONNEAU, B.; BOUGARAN, J. y cols.: "Prognosis significance of seminal vesicle invasion on the radical prostatectomy specimen". Eur. Urol., 33: 271, 1998.

**31. EPSTEIN, J.I.; PARTIN, A.W.; POTTER, S.R. y cols.: "Adenocarcinoma of the prostate invading the seminal vesicle: prognostic stratification based oh pathologic parameters". Urology, 56: 283, 2000.

32. D'AMICO, A.; WHITTINGTON, R.; MALKOWICZ, S.B. y cols.: "A multivariate analysis of clinical and pathologic factors that predict for prostate specific antigen failure after radical prostatectomy for prostate cancer". J. Urol., 154: 131, 1995

33. CATALONA, W.J.; SMITH, D.S.: "5-year tumor recurrence rates after anatomical radical retropubic prostatectomy for prostate cancer". J. Urol., 152: 1837, 1994.

34. EPSTEIN, J.I.; PARTIN, A.W.; SAUVAGEOT, J. y cols.: "Prediction of progression following radical prostatectomy: a multivariate analysis of 721 men with long-term follow-up". Am. J. Surg. Pathol., 20: 286, 1996.

35. TRAPASSO, J.G.; DE KERNION, J.B.; SMITH, R.B. y cols.: "The incidence and significance of detectable levels of serum prostate specific antigen after radical prostatectomy". J. Urol., 152: 1821, 1994.

*36. ZUDAIRE, J.J.; LÓPEZ, J.; SÁNCHEZ, D. y cols.: "Radical prostatectomy in stage pT3C stage prostatic adenocarcinoma". Actas Urol. Esp., 24: 468, 2000.

37. ROBERTS, W.W.; BERGSTRALH, E.J.; BLUTE, M.L. y cols.: "Contemporary identification of patiens at high risk of early prostate cancer recurrence after radical retropubic prostatectomy". Urology, 57: 1033, 2001.

38. BLUTE, M.L.; BERGSTRALH, E.J.; IOCCA, A. y cols.: "Use of Gleason score, prostate specific antigen, seminal vesicle and margin status to predict biochemical failure after radical prostatectomy". J. Urol., 165: 119, 2001.

39. LAU, W.K.; BLUTE, M.L.; BOSTWICK, D.G. y cols.: "Prognostic factors for survival of patients with pathological gleason score 7 prostate cancer: differences in outcome between primary gleason grades 3 and 4". J. Urol., 166: 1692, 2001.

*40. SOFER, M.; SAVOIE, M.; KIM, S.S. y cols.: "Biochemical and pathological predictors of the recurrence of prostatic adenocarcinoma with seminal vesicle invasion". J. Urol., 169: 153, 2003.

*41. BLOOM, K.D.; RICHIE, J.P.; SCHULTZ, D. y cols.: "Invasion of seminal vesicles by adenocarcinoma of the prostate: psa outcome determined by preoperative and postoperative factors". Urology, 63: 333, 2004.
*42. ALLEPUZ, C.A.; SANZ, J.I.; GIL, M.J. y cols.: "Seminal vesicle biopsy in prostate cancer staging". J. Urol., 154: 1407, 1995.

43. WALSH, P.C.: "Radical retropubic prostatectomy". Walsh PC, Retik AB, Stamey TA, Vaughan ED Jr, editores. Campbell's Urology. 6 ed. Philadelphia (PA): WB Saunders Company, p. 286586, 1992.

44. BLAS, M.; RIOJA, C.; GIL, M.J. y cols.: "Conferencia de consenso sobre Carcinoma de próstata". Madrid: ENE Ediciones, p. 81-92, 1996.

45. BLAS, M.; ALLEPUZ, C.; RIOJA, C. y cols.: "Valor y secuencia de la biopsia de vesículas seminales y la linfadenectomía pelviana laparoscópica en el estadificación del cáncer de próstata". Actas Urol. Esp., 21: 874, 1997.

**46. KASIBHATLA, M.; PETERSON, B.; ANSCHER, M.S.: "What is the best postoperative treatment for patients with pT3bN0M0 adenocarcinoma of the prostate?". Prostate Cancer and Prostatic Diseases, 8: 167, 2005.

47. NAKABAYASHI, M.; OH, W.K.: "Chemotherapy for high-risk localized prostate cancer". BJU Int., 97: 679, 2006.

48. J. STEPHENSON, A.J.; SCARDINO, P.T.; EASTHAM, J.A. y cols.: "Postoperative nomogram predicting the 10-year probability of prostate cancer recurrence after radical prostatectomy". J. Clin. Oncol., 23: 7005, 2005.

49. RIOJA, J.; ZUDAIRE, J.J.; RINCÓN, A. y cols.: "Adenocarcinoma de próstata gleason 8: influencia pronóstica en la supervivencia libre de progresión bioquímica". Actas Urol. Esp., 30: 117, 2006.

50. PARKER, C.; HAYCOCKS, T.; BAYLEY, A. y cols.: "A dose-volume histogram analysis of the seminal vesicles in men treated with conformal radiotherapy to "prostate alone". Clin. Oncl. (R. Coll. Radiol.), 14: 298, 2002.

51. KESTIN, L.; GOLDSTEIN, N.; VICINI, F. y cols.: "Treatment of prostate cancer with radiotherapy: should the entire seminal vesicles be included in the clinical target volume?". Int. J. Radiat. Oncol. Biol. Phys. 54: 686, 2002.

52. STOCK, R.G.; CAHLON, O.; CESARETTI, J.A. y cols.: "Combined modality treatment in the management of high-risk prostate cancer". BJU Int., 85: 281, 2000.

53. DE LA TAILLE, A.; BENSON, M.C.; BAGIELLA, E. y cols.: "Cryoablation for clinically localized prostate cancer using an argon-based system: complication rates and biochemical recurrence". BJU Int., 85: 281, 2000.

54. SALIKEN, J.C.; GRAY, R.R.; DONNELLY, B.J. y cols.: "Extraprostatic biopsy improves the staging of localized prostate cancer". Can. Assoc. Radiol. J., 51: 114, 2000. 